\title{
LuxS and quorum-sensing in Campylobacter
}

\author{
Paul J. Plummer ${ }^{1,2 *}$ \\ Department of Veterinary Diagnostic and Production Animal Medicine, College of Veterinary Medicine, lowa State University, Ames, IA, USA \\ 2 Department of Veterinary Microbiology and Preventative Medicine, College of Veterinary Medicine, lowa State University, Ames, IA, USA
}

\section{Edited by:}

Alain Stintzi, Ottawa Institute of

Systems Biology, Canada

Reviewed by:

Margaret E. Bauer, Indiana

University School of Medicine, USA

Martin R. Roop II, East Carolina

University, USA

\section{${ }^{*}$ Correspondence:}

Paul J. Plummer, 2426 Lloyd

Veterinary Medical Center, College of Veterinary Medicine, lowa State University, Ames, IA 50011, USA.

e-mail:pplummer@iastate.edu
Several intercellular bacterial communication mechanisms have been identified in a broad range of bacterial species. These systems, collectively termed quorum-sensing systems, have been demonstrated to play significant roles in a variety of bacterial processes including motility, biofilm formation, expression of virulence genes, and animal colonization. Campylobacter jejuni is known to possess a LuxS/ autoinducer-2 (Al-2) mediated system that have been partially characterized over the last decade. Al-2 is formed as a byproduct of the activated methyl recycling pathway, specifically by the LuxS enzyme. Previous work in our laboratory and that of others has demonstrated that this gene is involved in a variety of physiologic pathways of $C$. jejuni including motility, autoagglutination, cytolethal distending toxin (CDT) expression, flagellar expression, oxidative stress, and animal colonization. This review article will summarize the current research associated with LuxS in $C$. jejuni and will provide insights into the role of this system in the metabolism and intercellular communication of this organism. Additionally, the evidence for other quorum-sensing pathways in Campylobacter will be discussed.

Keywords: Campylobacter, quorum-sensing, Al-2, HSL, virulence

\section{GENERAL MECHANISMS OF OUORUM-SENSING}

The first evidence of bacterial communication came from the study of density-dependent bioluminescence of Vibrio fischeri (Engebrecht et al., 1983; Engebrecht and Silverman, 1984). The induction of bioluminescence was found to be mediated by the concentration-dependent action of small signaling compounds produced by the bacteria and termed autoinducers (AIs). This work provided the basis for the quorum-sensing model in Gram-negative bacteria where an acyl-homoserine lactone (HSL) autoinducer (AI-1) accumulates in the extracellular environment and freely diffuses into the bacterial cytoplasm. When intracellular AI levels reach a critical threshold concentration it binds to a cellular transcriptional activator (luxR homolog) that is responsible for luciferase gene induction (Bassler, 1999). Subsequent work has demonstrated a similar system in over 30 species of Gram-negative bacteria where it exerts control over a variety of cell density-dependent processes. In contrast, Gram-positive species have been demonstrated to produce small peptide signaling molecules that are secreted through the action of an $A B C$ transporter protein system (Bassler, 1999). The extracellular AIs are then recognized by a cognate two-component sensor kinase that initiates a phosphorelay signal transduction cascade. Perhaps most intriguing is the identification of a third quorum-sensing mechanism that appears to be highly conserved over a variety of gram-negative and gram-positive organisms. In these organisms the luxS gene is responsible for the synthesis of a novel AI termed autoinducer-2 (AI-2), which has mechanisms reminiscent of both the Gram-positive and Gram-negative systems. The model system, Vibrio harveyi, uses a well described two component sensor kinase system (LuxP/Q) to initiate a phosphorelay signal transduction event that ultimately leads to dephosphorylation of the response regulator ( $\mathrm{LuxO})$ and changes in gene expression
(Bassler et al., 1993). In contrast, Salmonella and, more recently, E. coli have both been demonstrated to rely on a periplasmic binding protein $(\mathrm{LsrB})$ that recognizes the $\mathrm{AI}-2$ and leads to internalization via an $\mathrm{ABC}$ transporter (encoded by the lsrACDBFGE operon) where the AI-2 is phosphorylated and regulates gene expression via LsrR (Taga et al., 2003; Xavier and Bassler, 2005b). According to genomic sequences, C. jejuni does not possess acylHSL synthetases and the AI-1 sensing system, but possesses a luxS homolog (Parkhill et al., 2000; Fouts et al., 2005). For the purposes of this review, we will first review what is known about LuxS and AI-2 production in C. jejuni since this has been the primary focus of quorum-sensing research in this species. Following the discussion of LuxS we will summarize the current understanding of alternative quorum-sensing mechanisms in Campylobacter, including the HSL/AI-1 system.

\section{LUXS AND AUTOINDUCER-2 PRODUCTION}

AI-2 production by the $S$-ribosylhomocysteinase, LuxS, was first described in Vibrio harveyi as a result of work on bioluminescence by this organism (Bassler et al., 1993). It was discovered that as the bacterial population grew, the environmental concentrations of AI-2 increased and that the presence of a critical concentration of this signal was necessary for bioluminescence of the organism. LuxS cleaves $S$-ribosylhomocysteine to form homocysteine and 4,5-dihydroxy-2,3-pentanedione (DPD) which is then spontaneously cyclized to form AI-2 (Winzer et al., 2002; De Keersmaecker et al., 2005; Rajan et al., 2005). As this system has been evaluated in a number of species it has become clear that the spontaneous cyclization of DPD does not produce a single molecular compound but instead can produce multiple molecules with varying abilities to bind AI-2 receptors. This has led to the use of the term "AI-2" to collectively represent a variety 
of molecular variants that result from spontaneous changes to DPD and act as ligands of AI-2 receptors (Vendeville et al., 2005). Recent work even suggests that these molecules are able to interconvert allowing for the inclusion of mixed bacterial populations in the quorum recognized by a single species (Xavier and Bassler, 2005a). The molecular form of AI-2 produced by C. jejuni is unknown at this time, however, work by our group and others groups has demonstrated that it is active as a luxP ligand during in vitro bioluminescence assays with a Vibrio harveyi reporter strain (Elvers and Park, 2002; Jeon et al., 2003; Quinones et al., 2009; Plummer et al., 2011b).

\section{THE STRUCTURE AND ACTIVITY OF LuXS}

Crystal structures of the LuxS homologs suggest that the protein forms a homodimer with a highly conserved active site (Vendeville et al., 2005). Structural studies have demonstrated that the LuxS protein forms a secondary structure containing a four-stranded antiparallel $\beta$ sheet interacting with four $\alpha$ helices (Hilgers and Ludwig, 2001; Lewis et al., 2001; Ruzheinikov et al., 2001). The LuxS structural fold observed in all of these crystals is highly conserved and appears to be a new type of fold in the alpha-beta family (Lewis et al., 2001). At the interface of the homodimer two identical active sites are present with a tetrahedrally coordinated $\mathrm{Fe}^{2+}$. Homodimerization also leads to the formation of two deep pockets adjacent to the active sites. The function of the pockets is still unclear, but they were demonstrated to hold a covalently bound homocysteine molecule in at least one study (Ruzheinikov et al., 2001). The proposed enzymatic activity involves a series of proton transfer reactions, catalyzed by two Lewis acid/bases and the divalent metal ion that will be discussed further in the next paragraph (Zhu et al., 2004). Although the LuxS protein of Campylobacter jejuni has not been crystallized, the gene sequence shows a high degree of homology to other sequenced strains $(74 \%$ aa identity to LuxS from Vibrio harveyi, $71 \%$ aa identity to LuxS from E. coli, and $73 \%$ aa identity to LuxS from Pasteurella multocida) and would be expected to have a similar structure (Elvers and Park, 2002).

LuxS is a $\mathrm{Fe}^{2+}$ metalloenzyme whose catalytic mechanism has been extensively studied and is well defined (Zhu et al., 2003; Pei and Zhu, 2004; Zhu et al., 2004; Rajan et al., 2005). Two universally conserved residues, Cys-84 and Glu-57 (amino acid numbering in E. coli LuxS), have been shown to act as critical general acids/bases during catalysis. In addition, several other active-site residues including Ser-6, His-11, and Arg-39 are also important for full activity of the LuxS enzyme (Pei and Zhu, 2004; Zhu et al., 2004). Recently we described the identification of a naturally occurring G92D mutation in some clones of C. jejuni 81116 (Plummer et al., 2011b). The glycine at the 92 position is invariant in enzymatically active enzymes described to date. Strains with this mutation fail to produce significant levels of AI2 in the biological systems utilized. Importantly, not all clones of 81116 harbor this mutation and several prior publications have demonstrated the presence of a functional LuxS enzyme and AI-2 production in this strain (Elvers and Park, 2002; Jeon et al., 2003). This finding underlines that significance of fully characterizing isolates used for quorum-sensing research even if they are strains previously reported in the literature to have functional LuxS systems.

\section{METABOLIC ROLE OF LuxS}

The primary function of LuxS in bacterial cells is as a critical component of the s-adenosyl-homocysteine recycling pathway. Functionally, LuxS is an s-adenosyl-homocysteinase responsible for hydrolysis of s-adenosylhomocysteine to homocysteine which is then further metabolized to reclaim s-adenosylmethionine (SAM). As a consequence, mutagenesis of LuxS results in both a loss of normal SAM recycling and a loss of AI-2 synthesis. This fact has greatly hindered the ability to specifically attribute any phenotypic differences associated with mutagenesis of LuxS with a specific loss of quorum-sensing ability. It is clear that mutagenesis of $l u x S$ results in alteration of the extracellular concentration of other SAM pathway metabolites. Using a recombinant LuxS protein bioassay we have demonstrated that the extracellular concentration of $s$-ribosylhomocysteine, the SAM intermediate on which LuxS functions, increases significantly in the culture supernatant of strains deficient in normal LuxS activity (Plummer et al., 2011b). Based on these findings, it is likely that many of the SAM pathway intermediates are present in altered concentrations both intracellularly and extracellularly in $\operatorname{luxS}$ mutant strains.

The SAM pathway is the primary means of methyl recycling in bacteria (Parveen and Cornell, 2011). Methyl groups donated by SAM are critical for the activity of methyl transferases that are intimately associated with bacterial DNA methylation, chemotaxis, motility, and a variety of other metabolic and biosynthetic reactions. SAM is also critical in bacterial polyamine formation and vitamin synthesis (Parveen and Cornell, 2011). As a consequence, alteration of SAM recycling associated with luxS mutagenesis can have significant impacts on bacterial metabolism.

\section{REGULATION OF LUXS EXPRESSION}

Hwang et al. (Hwang et al., 2011) recently reported that antisensemediated gene silencing of CosR, an essential response regulator of Campylobacter jejuni, upregulated LuxS expression by 1.7 fold. Similarly, over expression of the CosR protein resulted in a 2.2fold down regulation of LuxS expression. Collectively there results suggest that the CosR response regulator negatively regulates the expression of LuxS. Building on these findings the group was also able to demonstrate that CosR bindings to the promoter region of LuxS by showing that the incubation of labeled LuxS gene probes with recombinant CosR protein resulted in a concentrationdependent electrophoretic mobility shift of the probes. Finally, using DNase I fingerprinting methods the group was able to develop a consensus binding site for CosR, and they identified a putative binding site located 10 bp upstream of the LuxS start codon. Collectively, these studies provide strong evidence for CosR playing a role in the regulation of LuxS expression. As discussed later in this review, LuxS has been associated with the cellular response of Campylobacter to oxidative stress, thus additional studies focused on the role of CosR in this response are warranted.

\section{ENVIRONMENTAL SENSING OF AI-2}

Variation has been demonstrated in the mechanism of cellular recognition of environmental AIs. Recent evidence suggest that 
chemical interconversion of AI-2 may also allow the signal of one species to effect the response of another species (Xavier and Bassler, 2005b). At present, no mechanisms of cellular recognition of AI-2 are known for Campylobacter. In the initial report of AI-2 production by C. jejuni Elvers and Park (2002) showed that peak AI-2 concentrations were reached at $18 \mathrm{~h}$ and maintained at that level for at least $42 \mathrm{~h}$. This finding would suggest that Campylobacter does not have an active "LSR type" system that pumps the compound into the intracellular matrix. More recently, two studies have demonstrated that AI-2 supernatent concentrations peak in mid- to late-exponential growth and decline during stationary phase, more similar to the pattern observed in Salmonella and E. coli (Cloak et al., 2002; Quinones et al., 2009). Using a slightly different approach, Holmes et al. demonstrated that AI-2 was either inactivated or taken up by Campylobacter after the addition of exogenous AI-2 to cultures, although the levels of bioluminescence observed in the Vibrio bio assay remained similar to the levels produced endogenously by wildtype strains (Holmes et al., 2009). Collectively, these findings leave uncertainty regarding the fate of AI-2 in the culture supernatant of Campylobacter. There are no LSR homologs identified using comparative genomics, however, Campylobacter does encode a number of ABC type transporters that could be involved in uptake of AI-2. There is a need for additional work in this area to further define the mechanisms, if any, for sensing or uptake of AI-2 in this species.

\section{PHENOTYPES ASSOCIATED WITH LUXS MUTAGENESIS}

Alterations in biofilm formation, growth rates, antimicrobial production, motility, toxin secretion, and colonization have been observed with $\operatorname{luxS}$ mutants of different bacterial species (Vendeville et al., 2005). For an excellent review of the currently described phenotypic changes in more than 15 species see the paper by Vendeville et al. (Vendeville et al., 2005). The experimental design of many of these studies involves mutation of luxS and subsequent evaluation for phenotypic changes. As LuxS is required for both homocysteine production and AI-2 synthesis, the phenotypic changes associated with the luxS mutants could be due to AI-2-mediated quorum-sensing or the loss of metabolic function of LuxS. Most studies demonstrate the phenotypic changes in the presence of reduced AI-2 levels but fail to demonstrate the direct link of the AI presence with the phenotype. This issue of dissecting the significance of metabolic changes versus AI2 synthase activity (and the subsequent presence of AI- 2 in the environment) has been addressed by several authors and represents a significant challenge in this field of research (Vendeville et al., 2005; De Keersmaecker et al., 2006). A recent study in Listeria monocytogenes demonstrated that the increased biofilm thickness observed in $\operatorname{luxS}$ mutant strains may be associated with increased levels of s-ribosylhomocysteine in the media of the mutant strain presumably associated with the metabolic deficiency of the enzyme and not the lack of AI-2 (Challan Belval et al., 2006). Despite the shortcomings of some experimental designs at differentiating these roles, many of the observed phenotypic changes associated with AI-2 in other species involve essential molecular processes or virulence factors. As such, the findings remain scientifically significant and relevant regardless of the exact mechanisms for the phenotypic changes. The following sections will focus on a review of the current understanding of Campylobacter phenotypes associated with luxS mutagenesis. Table 1 includes a summary of phenotypes that have been associated with $\operatorname{lu} x S$ mutagenesis in this species.

\section{CONFIRMATION OF LUXS AND AUTOINDUCER-2 IN Campylobacter}

The publication of the first full genome sequence of $C$. jejuni in 2000 (Parkhill et al., 2000) allowed for the in silico identification of a luxS ortholog (Cj1198) in 2002 (Elvers and Park, 2002). Elvers and Park were able to demonstrate the presence of AI-2 activity in growth media of $C$. jejuni using the AI-2 bioluminesence assay reported by Bassler et al. (1993) and were able to eliminate the AI-2 activity by insertional mutation of the luxS gene. The authors were unable to demonstrate any differences in growth rate, resistance to oxidative stress, or in vitro invasion assays, but did show a decrease in motility of the mutant compared to the parent strain. Cloak et al. (2002) subsequently confirmed the presence of a functional $\operatorname{lu} x \mathrm{~S}$ gene and production of active AI2 using the same bioluminescence assay. Additionally, they were able to demonstrate measurable levels of AI-2 in milk and chicken broth inoculated with Campylobacter, suggesting that the nutrient requirements necessary for AI-2 are present in common food sources. Collectively these results provide strong evidence for the presence of a functional luxS gene capable of producing AI-2 in C. jejuni. Recently, Tazumi et al. (2011) screened 20 isolates of C. jejuni, C. coli, C. upsaliensis, C. fetus, and C. lari to determine how widely conserved the gene was in the genus. Interestingly, hybridization assays along with PCR demonstrated the presence of LuxS homologs in all isolates of C. jejuni, C. coli, C. upsaliensis, and C. fetus but failed to identify LuxS homologs in any of the four C. lari isolates. Furthermore, AI-2 bioluminescence assays showed the production of functional AI-2 products in all strains except the C. lari isolates. Based on these results it is clear that with the exception of C. lari, the majority of Campylobacter spp. have functional LuxS enzymes and produce AI-2.

\section{LuxS AND GROWTH}

Elvers and Park were the first group to evaluate the growth phenotypes of luxS mutants in C. jejuni. Using the NCTC 11168 strain they demonstrated that the mutant had a similar doubling time compared to the wildtype strain (103 min for the mutant compared to $110 \mathrm{~min}$ for the wildtype) (Elvers and Park, 2002). Furthermore, both strains required approximately $20 \mathrm{~h}$ to enter stationary phase. Subsequently, other studies have demonstrated essentially equal growth rates between the wildtype and LuxS mutant strains of C. jejuni 81116 (Jeon et al., 2003), M129 (Reeser et al., 2007), and NCTC 11168 grown in both Mueller Hinton broth and MEM- $\alpha$ broth(Holmes et al., 2009). Interestingly, $\mathrm{He}$ et al. (2008) showed that in C. jejuni 81-176 the LuxS mutant had a statistically longer doubling time compared to the wildtype strain when the isolates were incubated at $37^{\circ} \mathrm{C}$, but not at $42^{\circ} \mathrm{C}$ when the growth rates were not different. Recently another publication demonstrated that the LuxS mutant of the 81-176 strain had a decreased growth rate during the exponential phase but reached stationary phase at the same time as its wildtype counterpart when grown at $42^{\circ} \mathrm{C}$ (Quinones et al., 2009). Based on 
Table 1 | Campylobacter jejuni phenotypes associated with mutagenesis of LuxS.

\begin{tabular}{|c|c|c|}
\hline \multirow[t]{3}{*}{ Growth } & No significant difference & $\begin{array}{l}\text { Elvers and Park, } 2002 \\
\text { Jeon et al., } 2003 \\
\text { Reeser et al., } 2007 \\
\text { He et al., } 2008\end{array}$ \\
\hline & No difference at $42^{\circ} \mathrm{C}$ but decreased at $37^{\circ} \mathrm{C}$ & Holmes et al., 2009 \\
\hline & Decreased in exponential phase but reach stationary at the same time & Quinones et al., 2009 \\
\hline \multirow[t]{7}{*}{ Motility } & Decreased in luxS mutant & $\begin{array}{l}\text { Elvers and Park, } 2002 \\
\text { Jeon et al., } 2003 \\
\text { Holmes et al., } 2009 \\
\text { Quinones et al., } 2009 \\
\text { Plummer et al., } 2011 \text { b }\end{array}$ \\
\hline & Motility able to be complemented & Quinones et al., 2009 \\
\hline & & Plummer et al., 2011b \\
\hline & Motility decreased in mutant at $37^{\circ} \mathrm{C}$ but not at $42^{\circ} \mathrm{C}$ & He et al., 2008 \\
\hline & Motility not changed & Guerry et al., 2006 \\
\hline & Decrease in flaA transcription in mutant & Jeon et al., 2003 \\
\hline & & Holmes et al., 2009 \\
\hline \multirow[t]{5}{*}{ Host colonization } & Decreased colonization of chickens beyond day 4 post inoculation using 81-176 & Quinones et al., 2009 \\
\hline & Unchanged when monoinoculated NCTC 11168 & Plummer et al., 2011a \\
\hline & Decreased competitive fitness of mutant & Plummer et al., 2011a \\
\hline & Complete absence of colonization in chicks using IA3902 mutant & Plummer et al., 2011a \\
\hline & Complete absence of colonization in guinea pigs using IA3902 mutant & Plummer et al., 2011a \\
\hline \multirow[t]{2}{*}{ In vivo virulence } & Decreased survival of mutant in meat juice stored at $5^{\circ} \mathrm{C}$ & Ligowska et al., 2011 \\
\hline & $\begin{array}{l}\text { Complete loss of abortion phenotype in guinea pig abortion model using IA3902, } \\
\text { fully restored with complementation }\end{array}$ & Plummer et al., 2011a \\
\hline Biofilm formation & Decreased biofilm formation in mutant & Reeser et al., 2007 \\
\hline Cytolethal distending toxin & Decrease in cdt gene transcription & Jeon et al., 2005 \\
\hline \multirow[t]{2}{*}{ Autoagglutination } & Altered kinetics in mutant with decreased autoagglutination & Jeon et al., 2003 \\
\hline & Decreased autoagglutination & Guerry et al., 2006 \\
\hline \multirow[t]{2}{*}{ Cellular adherence and invasion } & No difference in wildtype and mutant on Caco-2 cells & Elvers and Park, 2002 \\
\hline & Decreased adherence and invasion of mutant on LMH cells & Quinones et al., 2009 \\
\hline \multirow[t]{4}{*}{ Oxidative stress } & No difference with hydrogen peroxide and paraquat & Elvers and Park, 2002 \\
\hline & Increased sensitivity of mutant to cumene hydroperoxide and hydrogen peroxide using & \\
\hline & disk diffusion & He et al., 2008 \\
\hline & Increased sensitivity of mutant to hydrogen peroxide using MIC method & He et al., 2008 \\
\hline \multirow[t]{2}{*}{ Chemotaxis } & Increased chemotaxis of mutant to amino acids & Quinones et al., 2009 \\
\hline & Decreased chemotaxis of mutant to organic acids & Quinones et al., 2009 \\
\hline
\end{tabular}

these collective results it appears that most strains of C. jejuni do not show significant growth defects associated with mutagenesis of LuxS enzyme, however, strain 81-176 seems to have additional complexity associated with the growth kinetics of mutant strains. Given that there are significant differences in how the bacteria were grown in each of these studies (Mueller-Hinton agar versus anaerobe basal agar, microaerophilic conditions established by Oxoid CampyGen versus anaerobic chamber) the reader should be cautious in trying to compare the differences observed between studies. In the case of 81-176, based on He et al. findings there was a temperature dependent difference in growth. Since the majority of other studies did not evaluate the growth kinetics under two temperatures in the same growth controlled growth conditions, it is not clear if similar differences might exist in other strains. Furthermore, not all of the studies (Elvers and Park, 2002; He et al., 2008) performed complementation of the mutant strains, thus secondary mutations associated with the changes in growth kinetics cannot be excluded.

\section{LuXS AND MOTILITY}

As mentioned previously, Elvers and Park (2002) provided the first experimental evidence for a role of LuxS in the motility of C. jejuni. Using semisolid media they demonstrated that the LuxS mutant formed a statistically smaller halo after incubation when compared to the wildtype strain. Subsequently, many studies have demonstrated that motility on semisolid media is decreased in luxS mutant strains (Jeon et al., 2003; Holmes et al., 2009; Quinones et al., 2009; Plummer et al., 2011b). In several cases this motility defect could be at least partially restored by genetic complementation of the luxS gene (Quinones et al., 2009; Plummer et al., 2011b). Similar to growth studies, C. jejuni strain 81-176 seems to show a temperature dependent difference in motility with a statistically significant decrease in the motility halo at $37^{\circ} \mathrm{C}$ but a non-statistically significant reduction in motility at $42^{\circ} \mathrm{C}$ (He et al., 2008). In contrast, a report of a luxS mutant of 81-176 generated to study autoagglutination demonstrated no loss of motility (Guerry et al., 2006). In that study, however, there is 
no genetic confirmation presented to demonstrate the construction of a loss of function mutant and no complementation studies were performed, thus the results should be interpreted with due caution.

In an attempt to explain the decreased motility of $\operatorname{luxS}$ null mutants Jeon et al. (2003) performed phase-contrast microscopy on the wildtype and luxS mutant strains and failed to observe any differences in motility speed or motility pattern. To study the molecular basis for the motility phenotype they evaluated transcription of the two major flagellin genes (flaA and flaB) using a primer extension assay. Using this assay they observed a 57\% reduction in the transcription of flaA in the luxS mutant background comparing to the wildtype strain. They were unable to demonstrate a difference in FlaA protein levels using western blotting and did not see a difference in flagellar structure based on transmission electron microscopy. Additionally, they were able to demonstrate decreased agglutination ability of the mutant strain. This change is presumably mediated by altered expression of surface proteins or flagella since a previous study has demonstrated a complete loss of agglutination in flagellar ablated strains (Golden and Acheson, 2002a). No significant changes were observed in the expression of flaB using the primer extension assay. Subsequently, Holmes et al. (2009) have confirmed a down regulation of flaA (4.2-fold down-regulated in luxS mutant compared to wildtype) using cDNA microarray.

\section{LUXS AND HOST COLONIZATION}

Two published reports have evaluated the role of LuxS in the colonization of the gastrointestinal system of animals. Quinones et al. (2009) utilized C. jejuni strain 81-176 to evaluate the colonization of the chicken gastrointestinal tract. They demonstrated a trend toward decreased colonization of the LuxS deficient strain starting at day 4 post inoculation and a statistically significant decrease of colonization of the mutant strain by 7 days post inoculation in two separate experiments. Although differences in mean colonization levels were observed there were several birds inoculated with mutant strain that maintained colonization levels similar to that observed in the wildtype throughout the study. The authors suggested that in these birds the exogenous levels of AI-2 produced by other enteric flora might be providing the support necessary to maintain colonization (Quinones et al., 2009). Our group has performed similar studies using the C. jejuni NCTC 11168 strain and the IA3902 strain of $C$. jejuni, a sheep abortion isolate extensively characterized by our group (Burrough et al., 2009; Sahin and Zhang, 2010). In our study, inoculation of chickens with mono-cultures of wildtype and $\operatorname{luxS}$ mutant strains of 11168 and the wildtype strain of IA3902 resulted in similar levels of colonization while the luxS mutant of IA3902 completely failed to colonize the gastrointestinal tract of chickens (Plummer et al., 2011a). A genetically complemented strain of the IA3902 carrying a chromosomally encoded luxS gene restored colonization to wildtype levels. Given that the LuxS deficient strain of 11168 colonized to normal levels, we proceeded to test this strain along with its wildtype counterpart in a competitive fitness experiment where we demonstrated a decreased fitness of the LuxS mutant associated with statistically significant decreases in recovery of the mutant compared to the wildtype populations (Plummer et al., 2011a).

We have also evaluated the role of LuxS in the colonization of the guinea pig gastrointestinal tract using strain IA3902. Following oral inoculation of the wildtype strain IA3902, its $\operatorname{luxS}$ mutant and a chromosomally encoded complement strain we saw a significant defect in the ability of the mutant strain to colonize the GI tract while the wildtype and complement strains showed high quantity and prolonged colonization based on fecal culture (Plummer et al., 2011a).

\section{LuXS AND VIRULENCE}

Very few studies have evaluated the role of LuxS in the virulence of C. jejuni. Using the IA3902 strain and the guinea pig model we have recently evaluated the role of LuxS in the virulence of this strain as a cause of abortion. When the wildtype IA3902 strain or its luxS mutant were inoculated by the intraperitoneal route into pregnant guinea pigs there was no difference observed in the abortion rates of the groups (Plummer et al., 2011a). In contrast, when the strains were inoculated by the oral route the $\operatorname{luxS}$ mutant strain was completely attenuated in its ability to produce abortions in the guinea pigs (5/9 aborted in wildtype versus $0 / 7$ in the mutant) (Plummer et al., 2011a). Furthermore, genetic complementation of the mutant strain resulted in full restoration of the abortion phenotype. Collectively these results suggest that LuxS plays an important role in the translocation of $C$. jejuni out of the intestine and into the systemic vasculature where it can move to the placenta and induce abortions.

\section{LuxS AND ENVIRONMENTAL ADAPTATION}

Ligowska et al. (2011) evaluated the survival of the NCTC 11168 strain in chicken meat juice incubated at $5^{\circ} \mathrm{C}$. They demonstrated that the luxS mutant of 11168 started decreasing significantly in number after six days of incubation while the wildtype numbers remained unchanged for the 27-day duration of the study. Based on these results they suggest that LuxS is important for the environmental adaptation to meat juice and cold temperatures.

\section{LUXS AND BIOFILM FORMATION}

In 2007, Reeser et al. demonstrated that $l u x S$ and flagellar structure may be important for C. jejuni biofilm growth since isolates that were mutagenized in either the flagellar proteins or $\operatorname{lu} x S$ had significantly decreased biofilm formation using a simple static biofilm assay. Using C. jejuni strain M129, a human clinical isolate, they demonstrated that the $\operatorname{luxS}$ mutant had a statistically significant reduction in crystal violet staining following a $48 \mathrm{~h}$ and $72 \mathrm{~h}$ incubation at $37^{\circ} \mathrm{C}$ in a $10 \% \mathrm{CO}_{2}$ environment. Given that similar reductions in crystal violet staining were observed in a fla $A B$ mutant it is unclear if the changes in biofilm formation were a direct response to the loss of a functional LuxS enzyme or secondary to altered transcription of flaA as demonstrated by Jeon et al. (2003). The overall significance of biofilms in the physiology and survival of $C$. jejuni is still not well understood, so the importance of these findings to bacterial survival or persistence in the environment is unclear presently. 


\section{LUXS AND CYTOLETHAL DISTENDING TOXIN}

Jeon et al. (2005) evaluated the role of AI-2 in expression of the cytolethal distending toxin (CDT), a putative virulence factor for C. jejuni. Using a primer extension assay they were able to demonstrate a $39 \%$ decrease in $c d t$ gene transcription when luxS was mutated. One biological function of CDT involves the arresting of the cell cycle in the $\mathrm{G}_{2} / \mathrm{M}$ phase (Whitehouse et al., 1998), and they were able to demonstrate a statistically significant decrease in number of cells arrested in this phase in the $\operatorname{luxS}$ mutant (Jeon et al., 2005). Recently, another study was published demonstrating that the expression of LuxS was up-regulated following incubation in chicken meat juices along with an up-regulation in the expression of CDT (Ligowska et al., 2011). It is unclear what mechanism underlies the up-regulation of CDT, however, the authors suggest that one possible mechanism would be through an up-regulation of LuxS expression. Additional work is necessary to further define the role of LuxS in the expression of CDT.

\section{LUXS AND AUTOAGGLUTINATION}

Jeon et al. (2003) also studied the role of $\operatorname{luxS}$ in autoagglutination of Campylobacter in an effort to determine if the mutation was associated with changes in surface properties exhibited by the bacteria. They were able to demonstrate a significant change in autoagglutination kinetics of the mutant strain compared to the wildtype, with the luxS mutant having a decreased autoagglutination. Similarly, Guerry et al. demonstrated that a luxS mutant of C. jejuni strain 81-176 had a decreased autoagglutination compared to the wildtype parent strains (Guerry et al., 2006). Autoagglutination has previously been associated with the presence of flagellar assemblies (Misawa and Blaser, 2000; Golden and Acheson, 2002b) and it is unclear if the observed change in autoagglutination is mediated by changes in flagellar assembly. Flagella are required for autoagglutionation, but are not sufficient by themselves to induce agglutination (Golden and Acheson, 2002b), therefore, other mechanisms of altered surface structure cannot be ruled out at this time.

\section{LuxS AND CELLULAR ADHERENCE AND INVASION}

In their initial description of the phenotypes associated with luxS mutagenesis, Elvers and Park (2002) failed to see a difference in adherence and invasion of Caco- 2 cells between the wildtype and mutant strains. In their study using C. jejuni NCTC 11168 they saw an internalization of $0.08 \%$ for the wildtype and $0.05 \%$ for the luxS mutant, which did not prove to be statistically different. More recently Quinones et al. evaluated the adherence of C. jejuni to LMH cells (Quinones et al., 2009). In their study they demonstrated a significant reduction $(P<0.01)$ in adherence of the luxS mutant of 81-176 when compared to the wildtype strain and that defect could be fully complemented with the addition of another luxS ORF. Given the variability of invasion phenotypes observed in C. jejuni, it is likely that the impact of luxS mutagenesis is determined by both the strain being tested and the eukaryotic cell type used for the assay. At present, no studies have evaluated the role of LuxS in the subvasion and invasion of eukaryotic monolayers, however, such experiments would likely provide useful information regarding the role of this gene in pathogenicity.

\section{LUXS AND OXIDATIVE STRESS}

Two independent studies have evaluated the role of LuxS in the cellular response to oxidative stress of $C$. jejuni. Elvers and Park (2002) used a well diffusion assay to test the oxidative stress of paraquat and hydrogen peroxide on C. jejuni NCTC 11168 and its luxS mutant. Using parquet concentrations between $1-40 \mathrm{mM}$ and hydrogen peroxide concentrations between 0.015 and $0.3 \%$ they could not demonstrate any differences in sensitivity (Elvers and Park, 2002). More recently He et al. compared the sensitivity of C. jejuni 81-176 and its $\operatorname{luxS}$ mutant to cumene hydroperoxide (CHP) and hydrogen peroxide using both a disk inhibition assay and a minimum inhibitory concentration (MIC) assay. Using the disk diffusion method they demonstrated a significant $(P$ ranging from $<0.001$ to $<0.0029$ ) increase in sensitivity to both compounds in a dose dependent fashion (He et al., 2008). Using the MIC methodology they found that MIC of the luxS mutant was two to four-fold lower than the MIC of the wildtype strain for hydrogen peroxide, consistent with the disk diffusion method. In contrast, the MIC of the luxS mutant and wildtype were the same for CHP. The group also demonstrated that the transcriptional expression of the $a p h C$ and $t p x$ genes, two stress response genes, appeared to be down regulated in the luxS mutant strain (He et al., 2008). The reason for the differences in response to hydrogen peroxide between the two studies is not clear. Neither study performed genetic complementation of the mutants to rule out secondary mutations and the methodologies used for testing the response were different (well diffusion in Elvers and Park versus disk diffusion/MIC in He et al.) making direct comparisons difficult.

\section{LUXS AND CHEMOTAXIS}

Quinones et al. used the hard-agar plug method to test the chemotactic response of $C$. jejuni 81-176 to both amino acids and organic acids. They demonstrated that zones of accumulation for the amino acids aspartate, asparagine, glutamate, and glutamine were all significantly increased in the luxS mutant strain compared to the wildtype (Quinones et al., 2009). They hypothesized that these changes might be a response to altered metabolic activity of the luxS mutant that resulted in disrupted amino acid metabolism and carbon compound catabolism. In contrast, the wildtype strain showed an increased chemotactic behavior for all tested organic acids when compared to the luxS mutant. Given that organic acids are important energy sources of $C$. jejuni in the chicken gastrointestinal tract they hypothesized that this decreased chemotactic activity may contribute to the decreased chicken colonization that they observed with this isolate.

\section{LUXS AND TRANSCRIPTIONAL REGULATION}

Two studies have been published that evaluated the role of LuxS mutagenesis in transcriptional regulation of $C$. jejuni (He et al., 2008; Holmes et al., 2009). Both studies performed a cDNA microarray analysis comparing the mRNA levels of wildtype versus luxS mutant. Furthermore, He et al. explored the role of LuxS in the presence of hydrogen peroxide used to induce oxidative stress while Holmes et al. evaluated transcriptional changes associated with the addition of exogenous AI-2. The results of both studies demonstrate altered expression of several flagellar, 
SAM metabolism, and ABC transporter genes in the luxS mutant, which would be consistent with the previously published phenotypes. Interestingly, $\mathrm{He}$ et al. observed that the mutant strain was more sensitive to the toxic effects of hydrogen peroxide and CHP. This phenotype was confirmed by altered expression of both the $a h p C$ (encoding alkyl hydroperoxide reductase) and tpx (encoding thiol peroxidase) genes (He et al., 2008). Collectively these findings would suggest that the $\operatorname{luxS}$ gene or AI-2 plays a role in the response of $C$. jejuni to oxidative stress. During this study they did not compare the differential transcriptional regulation of C. jejuni luxS mutants in the presence or absence of exogenous AI2 , so it is difficult to dissect how much of the observed change was associated with the metabolic role of $l u x S$ as opposed to its role in AI-2 synthesis. More recently, Holmes et al. reported a microarray experiment designed to further evaluate the issue of AI-2 sensing by C. jejuni. In their experiment they added exogenous AI-2 to the culture media of luxS mutant strains and evaluated transcriptional changes in gene expression. They were unable to find a significant number of genes that were differentially expressed in the presence of exogenous AI-2 and concluded that most of the transcriptional changes observed in mid-exponential growth luxS mutants of C. jejuni were the result of metabolic disturbances induced by altering the SAM recycling pathway. They concluded that AI-2 mediated quorum-sensing is not an important mechanism in mid-exponential growth of C. jejuni cultured in $\mathrm{MH}$ media (Holmes et al., 2009). Interestingly, they also performed the microarray analysis on samples recovered following a minimal media (MEM- $\alpha$ as opposed to $\mathrm{MH}$ broth) incubation. They demonstrate that there was a media dependent association of gene expression with almost twice as many genes being differentially expressed in the complex media versus the minimal media (131 vs. 60 genes for the MHB media and MEM media, respectively) (Holmes et al., 2009). Of those genes there was a subset of 20 genes that were differentially expressed in both media types.

In general, transcriptional changes in C. jejuni luxS mutants are less extensive then those demonstrated in other bacterial species. For instance, LuxS appears to act as a global regulator of E. coli where 5-10\% of the genes in the genome are expressed differentially in the mutant compared to wildtype (Delisa et al., 2001; Sperandio et al., 2001). In C. jejuni less than $2 \%$ of the genes are differentially regulated suggesting that LuxS is not a global regulator in this species (He et al., 2008; Holmes et al., 2009).

\section{OTHER QUORUM-SENSING COMPOUNDS IN C. jejuni AUTOINDUCER-1 AND Campylobacter}

As mentioned previously $C$. jejuni does not encode obvious homologs of AI-1 synthase genes. Moorhead et al. performed a BLASTN search against C. jejuni strains 11168 and RM1221 using the $l u x I$ and ainS synthase genes as queries and did not identify any putative homologs (Moorhead and Griffiths, 2011). However, the sequence similarity of these genes has been reported to be very low, often with values lower than 35\% for pair-wise identity, making identification difficult (Fuqua et al., 1996). Using a screen of eight AI-2 biosensors they were able to demonstrate that two C. jejuni strains (81-176 and cj11) both produced positive reactions in the $V$. harveyi $\mathrm{BB} 886$ sensor system. This result suggests that these strains are able to produce an HSL (AI-1) product similar to $\mathrm{N}$-(3-hydroxy-butanoyl)-L-HSL (3-OH-C4HSL) (Moorhead and Griffiths, 2011). The investigators were able to fractionate the samples and obtain a purified fraction that demonstrated the biological activity in the HSL sensor bioassay. They named this fraction cjA. Subsequently, they tested a panel of six different HSLs and cjA for their ability to induce changes in the formation of a viable but non-culturable state (VBNC), biofilm formation, virulence gene transcription, and IL-8 stimulation of INT-407 cells. In summary, the short chained HSLs, along with cjA prolonged the onset of the VBNC state although the difference was fairly small. Likewise the addition of 3-OH-C4-HSL or cjA disrupted the ability of strain $81-176$ to form microscopic biofilms evaluated using confocal laser scanning microscopy (CLSM) while the addition of the five other HSL compounds had minimal impact. Using RT-PCR the authors then evaluated the expression of five previously described virulence genes associated with $C$. jejuni pathogenesis. Included in the study were $c a d F$, $c d t B, c i a B, f l a A$, and iamA. The additional of several of the HSL compounds resulted in significant changes in transcriptional levels of these virulence genes with the cjA fraction purified from C. jejuni cell-free media inducing a 7-fold up-regulation of $c a d F$, a 6.60 -fold increase in $c d t B$, a 1.98 -fold increase in $c i a B$, a 2.27 fold increase in flaA and a 10.0-fold decrease in iamA. Collectively these results suggest that $C$. jejuni is able to either produce a HSL compound or a mimic that is capable of inducing a response in bioassays for C4-HSL. Furthermore, the differences in biofilm formation and gene expression following exogenous addition of cjA and other HSL compounds suggest that the organism is capable of sensing and responding to these compounds in the environment. Additional work is necessary to define the role of HSL signaling in C. jejuni and to determine the molecular mechanisms responsible for the synthesis and sensing of these compounds.

\section{AUTOINDUCER-3 AND Campylobacter}

Work conducted by Sperandio et al. (2003) demonstrated that in vitro synthesized AI-2 was unable to induce the expression of the locus of enterocyte effacement (lee) in E. coli and did not complement the type III secretion system phenotype observed in EHEC luxS mutants. Furthermore, they demonstrated that using column purification they could induce lee expression and restore the type III secretion system with a purified fraction different from the fraction exhibiting AI-2 activity by the Vibrio bioluminescence assay. They associated this activity with a new AI named AI-3 and developed a LEE1::lacZ fusion for assaying cell free supernatents for the presence of this compound. Importantly, mutation of the $l u x S$ gene in E. coli leads to the loss of both AI-2 and AI-3 activity (52). Testing of Campylobacter cell free supernatants for the presence of AI-3 using the LEE1::lacZ fusion kindly provided by Dr. V. Sperandio have failed to demonstrate such activity in Campylobacter (unpublished data from our laboratory).

\section{CONCLUSIONS}

It is clear from multiple studies that the mutagenesis of the LuxS enzyme in C. jejuni results in repeatable phenotypes, some of which can significantly alter its virulence. Based on the dual function of LuxS as both a key metabolic enzyme in the SAM recycling 
pathway and the synthase protein for the precursor of AI-2 it is more difficult to determine which of these mechanisms underlies the phenotypic changes. Holmes et al. have argued that the lack of a transcriptional response to exogenous AI-2 would suggest that Campylobacter is not readily able to recognize and respond to AI2 as a quorum-sensing molecule in the conditions tested (Holmes et al., 2009). We must consider the fact that the conditions tested were significantly different from the microenvironments present in natural host and as such do not exclude the role of AI-2 as a sensing compound in other environments.

\section{REFERENCES}

Bassler, B. L. (1999). How bacteria talk to each other: regulation of gene expression by quorum sensing. Curr. Opin. Microbiol. 2, 582.

Bassler, B. L., Wright, M., Showalter, R. E., and Silverman, M. R. (1993). Intercellular signaling in Vibrio harveyi: sequence and function of genes regulating expression of luminescence. Mol. Microbiol. 9, 773-786.

Burrough, E. R., Sahin, O., Plummer, P. J., Zhang, Q., and Yaeger, M. J. (2009). Pathogenicity of an emergent, ovine abortifacient Campylobacter jejuni clone orally inoculated into pregnant guinea pigs. Am. J. Vet. Res. 70, 1269-1276.

Challan Belval, S., Gal, L., Margiewes, S., Garmyn, D., Piveteau, P., and Guzzo, J. (2006). Assessment of the roles of LuxS, S-ribosyl homocysteine, and autoinducer 2 in cell attachment during biofilm formation by Listeria monocytogenes EGD-e. Appl. Environ. Microbiol. 72, 2644-2650.

Cloak, O. M., Solow, B. T., Briggs, C. E., Chen, C. Y., and Fratamico, P. M. (2002). Quorum sensing and production of autoinducer-2 in Campylobacter spp., Escherichia coli O157:H7, and Salmonella enterica serovar Typhimurium in foods. Appl. Environ. Microbiol. 68, 4666-4671.

De Keersmaecker, S. C. J., Sonck, K., and Vanderleyden, J. (2006). Let LuxS speak up in AI-2 signaling. Trends Microbiol. 14, 114.

De Keersmaecker, S. C. J., Varszegi, C., van Boxel, N., Habel, L. W., Metzger, K., Daniels, R., Marchal, K., De Vos, D., and Vanderleyden, J. (2005). Chemical synthesis of (S)4,5-Dihydroxy-2,3-pentanedione, a bacterial signal molecule precursor, and validation of its activity in Salmonella typhimurium. J. Biol. Chem. 280, 19563-19568.

Delisa, M. P., Wu, C. F., Wang, L., Valdes, J. J., and Bentley, W. E. (2001). DNA microarray-based identification of genes controlled by autoinducer 2-stimulated quorum sensing in Escherichia coli. J. Bacteriol. 183, 5239-5247.

Elvers, K. T., and Park, S. F. (2002). Quorum sensing in Campylobacter jejuni: detection of a luxS encoded signalling molecule. Microbiology 148, 1475-1481.

Engebrecht, J., Nealson, K., and Silverman, M. R. (1983). Bacterial bioluminescence: isolation and genetic analysis of functions from Vibrio fischeri. Cell 32, 773-781.

Engebrecht, J., and Silverman, M. R. (1984). Identification of genes and gene products necessary for bacterial bioluminescence. Proc. Natl. Acad. Sci. U.S.A. 81, 4154-4158.

Fouts, D. E., Mongodin, E. F., Mandrell, R. E., Miller, W. G., Rasko, D. A., Ravel, J., Brinkac, L. M., Deboy, R. T., Parker, C. T., Daugherty, S. C., Dodson, R. J., Durkin, A. S., Madupu, R., Sullivan, S. A., Shetty, J. U., Ayodeji, M. A., Shvartsbeyn, A., Schatz, M. C., Badger, J. H., Fraser, C. M., and Nelson, K. E. (2005). Major structural differences and novel potential virulence mechanisms from the genomes of multiple Campylobacter Species. PLoS Biol. 3:e15. doi: 10.1371/journal. pbio. 0030015

Fuqua, C., Winans, S. C., and Greenberg, E. P. (1996). Census and consensus in bacterial ecosystems: the LuxR-LuxI family of quorumsensing transcriptional regulators. Ann. Rev. Microbiol. 50, 727-751.

Golden, N., and Acheson, D. (2002a). autoaggultination Campylobacter jejuni mutants by random transposon mutagenesis. Infect. Immun. 70, 1761-1771.

Golden, N. J., and Acheson, D. W. (2002b). Identification of motility and autoagglutination Campylobacter jejuni mutants by random transposon mutagenesis. Infect. Immun. 70, 1761-1771.

Guerry, P., Ewing, C. P., Schirm, M., Lorenzo, M., Kelly, J., Pattarini, D. Majam, G., Thibault, P., and Logan, S. (2006). Changes in flagellin glycosylation affect Campylobacter Identification of motility and

The recent identification of a putative HSL type compound produced by $C$. jejuni demonstrates that one cannot rely exclusively on comparative genomics as a means of studying quorumsensing in this species. There remain a lot of significant questions regarding this potential HSL compound including: What gene is responsible for production of cjA? How the product is sensed? Are the observed changes in phenotype specific to the compound? Future work in these areas will provide critical information regarding the role of this compound in the pathogenesis of C. jejuni.

autoagglutination and virulence. Mol. Microbiol. 60, 299-311.

He, Y., Frye, J. G., Strobaugh, T. P., and Chen, C. Y. (2008). Analysis of AI2/LuxS-dependent transcription in Campylobacter jejuni strain 81-176. Foodborne Pathog. Dis. 5, 399-415.

Hilgers, M. T., and Ludwig, M. L. (2001). Crystal structure of the quorum-sensing protein LuxS reveals a catalytic metal site. Proc. Natl. Acad. Sci. U.S.A. 98, 11169-11174.

Holmes, K., Tavender, T. J., Winzer, K., Wells, J. M., and Hardie, K. R. (2009). AI-2 does not function as a quorum sensing molecule in Campylobacter jejuni during exponential growth in vitro. BMC Microbiol. 9, 214.

Hwang, S., Kim, M., Ryu, S., and Jeon, B. (2011). Regulation of oxidative stress response by CosR, an essential response regulator in Campylobacter jejuni. PloS One 6:e22300. doi: 10.1371/journal.pone.0022300

Jeon, B., Itoh, K., Misawa, N., and Ryu, S. (2003). Effects of quorum sensing on flaA transcription and autoagglutination in Campylobacter jejuni. Microbiol. Immunol. 47, 833-839.

Jeon, B., Itoh, K., and Ryu, S. (2005). Promoter analysis of cytolethal distending toxin genes $(\operatorname{cdt} \mathrm{A}, \mathrm{B}$, and C) and effect of a luxS mutation on CDT production in Campylobacter jejuni. Microbiol. Immunol. 49, 599-603.

Lewis, H. A., Furlong, E. B., Laubert, B., Eroshkina, G. A., Batiyenko, Y., Adams, J. M., Bergseid, M. G., Marsh, C. D., Peat, T. S., and Sanderson, W. E. (2001). A structural genomics approach to the study of quorum sensing: crystal structures of three LuxS orthologs. Structure 9, 527.

Ligowska, M., Cohn, M. T., Stabler, R. A., Wren, B. W., and Brondsted, L. (2011). Effect of chicken meat environment on gene expression of Campylobacter jejuni and its relevance to survival in food. Int. J. Food Microbiol. 145(Suppl. 1), S111-S115.
Misawa, N., and Blaser, M. J. (2000). Detection and characterization of autoagglutination activity by Campylobacter jejuni. Infect. Immun. 68, 6168-6175.

Moorhead, S. M., and Griffiths, M. W. (2011). Expression and characterization of cell-signalling molecules in Campylobacter jejuni. J. Appl. Microbiol. 110, 786-800.

Parkhill, J., Wren, B. W., Mungall, K., Ketley, J. M., Churcher, C., Basham, D., Chillingworth, T., Davies, R. M., Feltwell, T., Holroyd, S., Jagels, K., Karlyshev, A. V., Moule, S., Pallen, M. J., Penn, C. W., Quail, M. A., Rajandream, M. A., Rutherford, K. M., Van Vliet, A. H. M., Whitehead, S., and Barrell, B. G. (2000). The genome sequence of the food-borne pathogen Campylobacter jejuni reveals hypervariable sequences. Nature 403, 665.

Parveen, N., and Cornell, K. A. (2011). Methylthioadenosine/S-adenosylhomocysteine nucleosidase, a critical enzyme for bacterial metabolism. Mol. Microbiol. 79, 7-20.

Pei, D., and Zhu, J. (2004). Mechanism of action of Sribosylhomocysteinase (LuxS). Curr. Opin. Chem. Biol. 8, 492.

Plummer, P., Sahin, O., Burrough, E. Sippy, R., Mou, K., Rabenold, J., Yaeger, M., and Zhang, Q. (2011a). The critical role of LuxS in the virulence of Campylobacter jejuni in a guinea pig model of abortion. Infect. Immun. 80, 585-593.

Plummer, P., Zhu, J., Akiba, M., Pei, D., and Zhang, Q. (2011b). Identification of a key amino acid of LuxS involved in AI-2 production in Campylobacter jejuni. PloS One 6:e15876. doi: 10.1371/journal. pone. 0015876

Quinones, B., Miller, W. G., Bates, A. H., and Mandrell, R. E. (2009). Autoinducer-2 production in Campylobacter jejuni contributes to chicken colonization. Appl. Environ. Microbiol. 75, 281-285.

Rajan, R., Zhu, J., Hu, X., Pei, D., and Bell, C. (2005). Crystal structure of S-ribosylhomocysteinase (LuxS) 
in complex with a catalytic 2ketone intermediate. Biochemistry 44, 3745-3753.

Reeser, R. J., Medler, R. T., Billington, S. J., Jost, B. H., and Joens, L. A. (2007). Characterization of Campylobacter jejuni biofilms under defined growth conditions. Appl. Environ. Microbiol. 73, 1908-1913.

Ruzheinikov, S. N., Das, S. K., Sedelnikova, S. E., Hartley, A., Foster, S. J., Horsburgh, M. J., Cox, A. G., McCleod, C. W., Mekhalfia, A., and Blackburn, G. M. (2001). The 1.2 A structure of a novel quorum-sensing protein, Bacillus subtilis LuxS. J. Mol. Biol. 313, 111.

Sahin, O., and Zhang, Q. (2010). Complete Genome Sequence of a highly virulent Campylobacter jejuni strain associated with Sheep Abortion in the United States. http://www.ncbi.nlm.nih. gov/nuccore/284925303.

Sperandio, V., Torres, A. G., Giron, J. A., and Kaper, J. B. (2001). Quorum sensing is a global regulatory mechanism in enterohemorrhagic
Escherichia coli O157:H7. J. Bacteriol. 183, 5187-5197.

Sperandio, V., Torres, A. G., Jarvis, B., Nataro, J. P., and Kaper, J. B. (2003). Bacteria-host communication: the language of hormones. PNAS 15, 8951-8956.

Taga, M. E., Miller, S. T., and Bassler, B. L. (2003). Lsr-mediated transport and processing of AI-2 in Salmonella typhimurium. Mol. Microbiol. 50, 1411-1427.

Tazumi, A., Negoro, M., Tomiyama, Y., Misawa, N., Itoh, K., Moore, J. E., Millar, B. C., and Matsuda, M. (2011). Uneven distribution of the luxS gene within the genus Campylobacter. Br. J. Biomed. Sci. 68, 19-22.

Vendeville, A., Winzer, K., Heurlier, K., Tang, C. M., and Hardie, K R. (2005). Making "sense" of metabolism: autoinducer-2, LuxS and pathogenic bacteria. Nat. Rev. Microbiol. 3, 383.

Whitehouse, C. A., Balbo, P. B., Pesci, E. C., Cottle, D. L., Mirabito, P. M., and Pickett, C. L. (1998). Campylobacter jejuni cytolethal distending toxin causes a G2-phase cell cycle block. Infect. Immun. 66, 1934-1940.

Winzer, K., Hardie, K. R., Burgess, N., Doherty, N., Kirke, D., Holden, M. T. G., Linforth, R., Cornell, K. A., Taylor, A. J., Hill, P. J., and Williams, P. (2002). LuxS: its role in central metabolism and the in vitro synthesis of 4-hydroxy-5-methyl3(2H)-furanone. Microbiology 148, 909-922.

Xavier, K. B., and Bassler, B. L. (2005a). Interference with AI-2mediated bacterial cell-cell communication. Nature 437, 750.

Xavier, K. B., and Bassler, B. L. (2005b). Regulation of uptake and processing of the quorum-sensing autoinducer AI-2 in Escherichia coli. J. Bacteriol. 187, 238-248.

Zhu, J., Dizin, E., Hu, X., Wavreille, A. S., Park, J., and Pei, D. (2003) $S$-Ribosylhomocysteinase (LuxS) is a mononuclear iron protein. Biochemistry 42, 4717-4726.

Zhu, J., Patel, R., and Pei, D. (2004). Catalytic Mechanism of
S-Ribosylhomocysteinase (LuxS): stereochemical course and kinetic isotope effect of proton transfer reactions. Biochemistry 43, 10166-10172.

Conflict of Interest Statement: The author declares that the research was conducted in the absence of any commercial or financial relationships that could be construed as a potential conflict of interest.

Received: 28 October 2012; accepted: 14 February 2012; published online: 06 March 2012.

Citation: Plummer PJ (2012) LuxS and quorum-sensing in Campylobacter. Front. Cell. Inf. Microbio. 2:22. doi: 10.3389/fcimb.2012.00022

Copyright (c) 2012 Plummer. This is an open-access article distributed under the terms of the Creative Commons Attribution Non Commercial License, which permits non-commercial use, distribution, and reproduction in other forums, provided the original authors and source are credited. 